\title{
TECHNOLOGICAL ISSUES OF STORING DYNAMIC DATA IN A RELATIONAL DATABASE ON RESEARCH PROJECTS
}

\author{
P. Milev* \\ University of National and World Economy, Sofia, Bulgaria
}

\begin{abstract}
The paper examines the possibility of storing dynamic data in a relational database. Under dynamic data the paper refers to data with variable structure and variable nature of different periods in terms of research projects. The relational model of databases, which is used everywhere, presents each entity of the system in a separate table. Reasonable, relationships and dependencies between them are described by defining the relationship between the relational tables. Thus, changes in nomenclatures which would lead to changes in the databases of information system will cause the need for new fields in relational tables, or even new tables and relationships that will inevitably lead to changes in software solutions. The paper focuses on how we can reorganize the data in the system, so changes in the database due to changes in database structure to be minimal. The aim of this paper is to examine the way the data for research projects is stored within the database. Specific for the data is that it has a different structure for each calendar year. For this reason, a relational database should often change its structure in order to determine proper functioning of the system. The paper presents a method of storing data on the principle of attribute - value, where the software part cares for the integrity of the data and the database takes care only for the storage. The paper presents a model for such a dynamic storage in a relational database and examines its advantages and disadvantages.
\end{abstract}

Key words: dynamic data, relational database, research projects.

\section{INTRODUCTION}

Nowadays all the stages of operation of research projects uses computer based information systems that automate the actions of application, evaluation, negotiation and reporting of research projects. Its operation goes through the stages, illustrated in Figure 1.

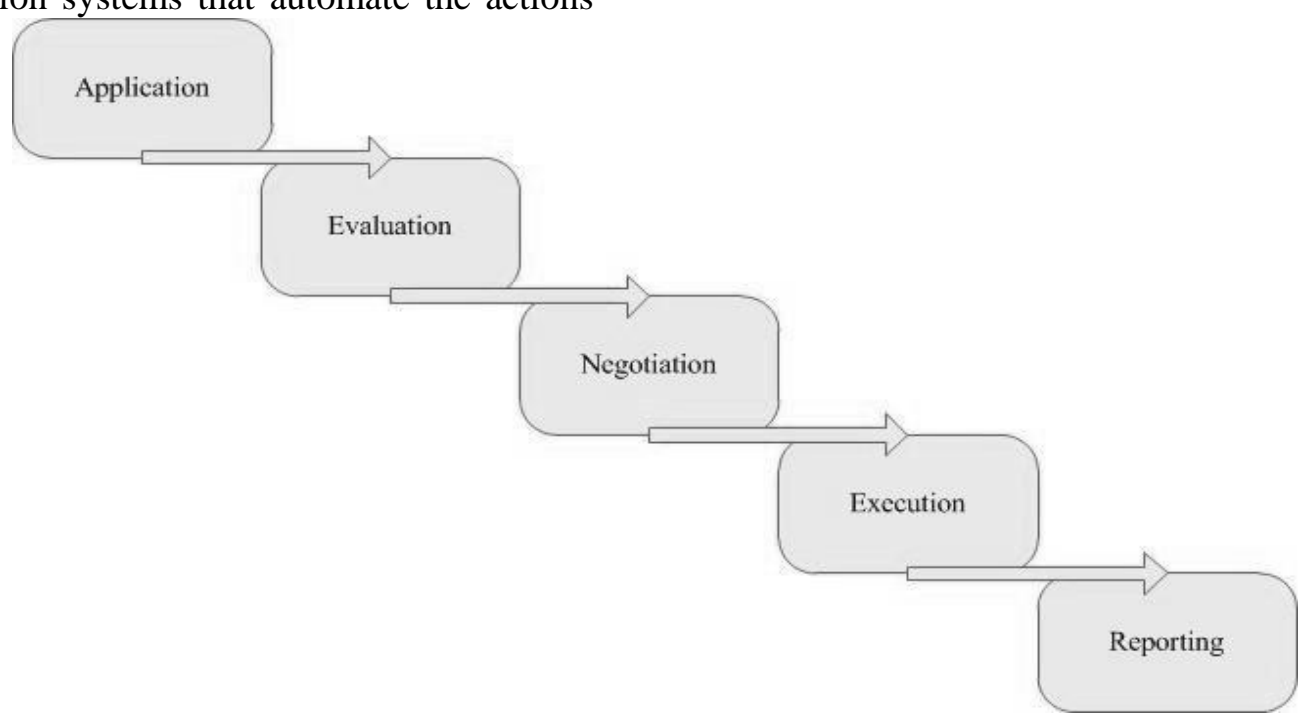

Figure 1. Stages of operation of the research projects

\footnotetext{
*Correspondence to: Plamen Milev, University of National and World Economy, Sofia 1700, tel. +35928195 312, e-mail: 
Stages of operation of research projects as a part of public projects and university projects are discussed in the publications by other authors $(1,2)$. Usually there are four different types of users within the information systems for research projects, illustrated in Figure 2:

- Administrators - these are users, who should be able to modify all major nomenclatures and should be able to configure the system to work according to the budget allocation. These users are responsible for implementation of all documents related to the project during its life cycle. It is possible for the administrators to obtain information, monitor and inform other users about expiring terms on certain tasks where they
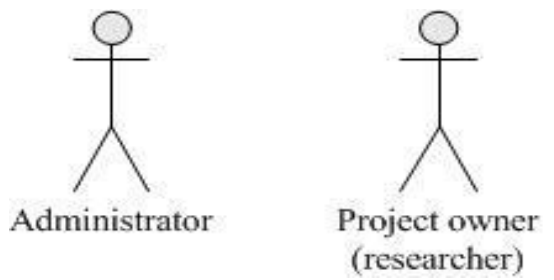

are overdue. Administrators have rights for preparation of reports and statements.

- Project owners (researchers) - users of this role have the rights to submit project proposals and for each stage of the project to introduce and edit data in the project. All the adjustments and corrections are only possible within the research regulations.

- Reviewers - these users have access to certain projects and possess the possibility to give reviews, including text reasoning, numerical estimates and conclusions on the projects.

- Auditors - users of this role have access to all the records in project proposals and also to the reviews of the projects. Their rights include rating and ranking of the projects, negotiation and acceptance of reports.

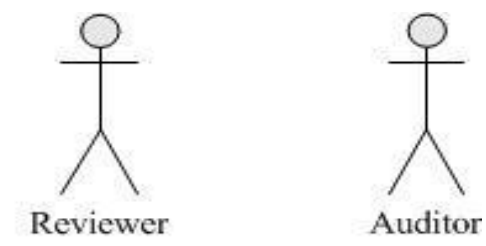

Figure 2. Types of users within a system for research projects

At each of these stages the information system works with different data. The aim of this paper is to examine the way the data for research projects is stored within the database. Specific for the data is that it has a different structure for each calendar year. For this reason, a relational database should often change its structure in order to determine proper functioning of the system. The paper presents a method of storing data on the principle of attribute - value, where the software part cares for the integrity of the data and the database takes care only for the storage.

\section{RELATIONAL DATA WITHIN THE DATABASE}

According A portion of the database is for a description of standard nomenclatures. These include:

- Researchers - they are characterized by scientific degree, academic position, organization, full name, email, phone and address;

- Scientific degrees - each researcher can have a scientific degree. Scientific degree is characterized by name and short name (abbreviation);

- Academic positions - each researcher can take an academic position. Academic position is characterized by name and short name (abbreviation);

- Organization - each researcher belongs to a scientific organization. Organization is characterized by name and address;

- Login accounts - each researcher has a login account in order to access the information system. Login account is characterized by username, password and role.

This part of the database is illustrated graphically in Figure 3. It consists of five tables with four relations between them. That is the right way for the description of these nomenclatures, because they are constant in the time chart. In this case, the storage of data in a standard relational database is more than adequate. If necessary, it is possible additional fields in any of the tables to be added, which is unlikely to have often. The illustrated database schema complies with the rules for the design of relational databases and complies with the rules of normalization of database.

So created tables assume the existence of standard program code for communication with the database and extraction of data from respective tables. Possible changes in the structure of these tables will surely lead to a change in the program code of the information system. 


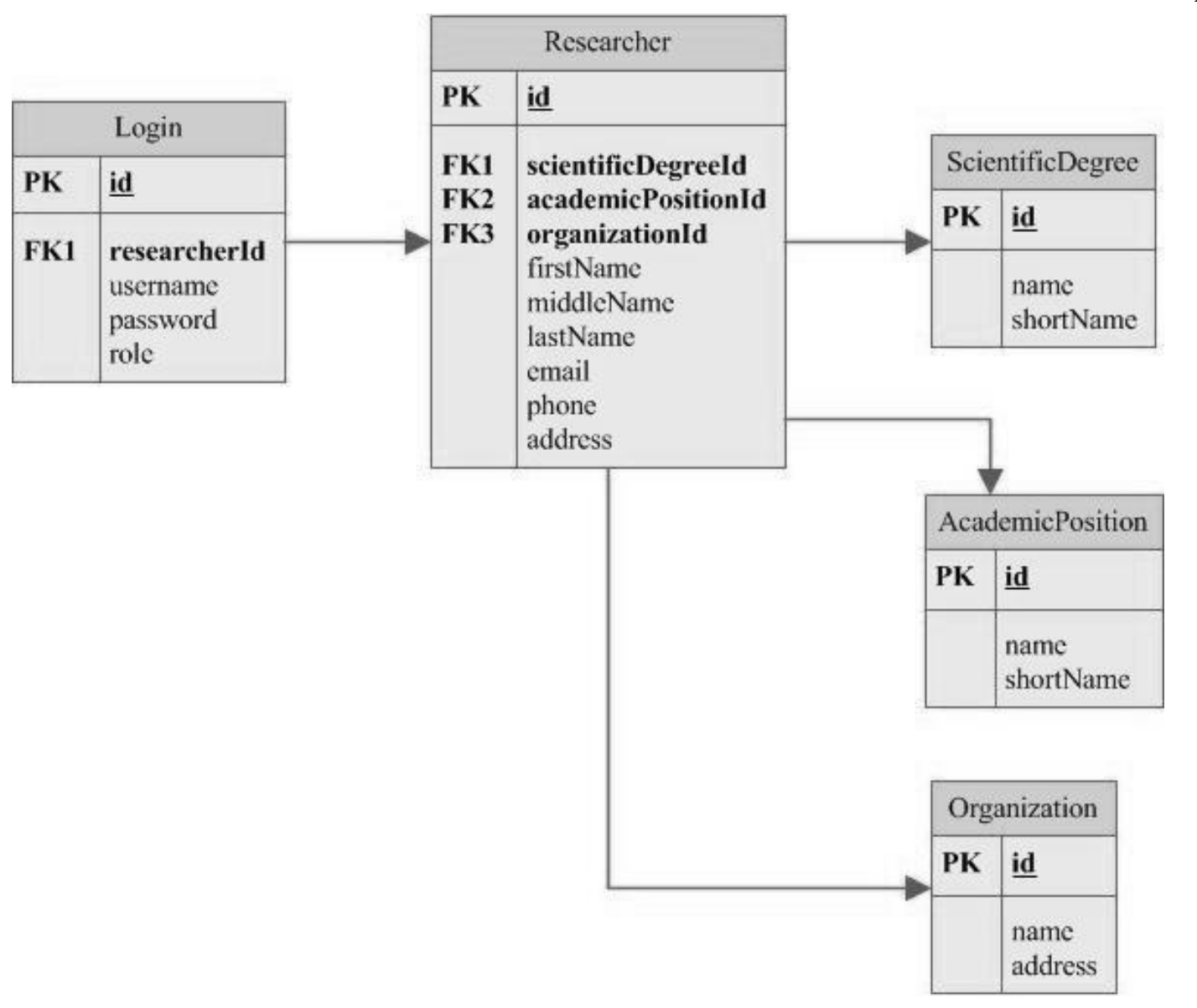

Figure 3. Data model for the relational part of the database

\section{DYNAMIC DATA WITHIN THE RELATIONAL DATABASE}

The relational model of databases, which is used everywhere, presents each entity of the system in a separate table. Reasonable, relationships and dependencies between them are described by defining the relationship between the relational tables. Thus, changes in nomenclatures which would lead to changes in the databases of information system will cause the need for new fields in relational tables, or even new tables and relationships that will inevitably lead to changes in software solutions. Here we focus on how we can reorganize the data in the system, so changes in the database due to changes in database structure to be minimal. Let's take a look at Figure 4 . We are trying to illustrate a database schema, which we will call an N-ISAV-Ref model. This schema is based on the so-called Entity-attribute-value (EAV) model (3). EAV is a model that describes the data entities, where the number of attributes that can be used to describe them is potentially huge, but the number that actually presents attributes for a particular substance is relatively modest. Data in the EAV model is shown in three columns. Entity identifies the elements. Attribute uniquely describes a characteristic of the entity. Value contains the value of this attribute. In this sense, we will call the model at Figure $\mathbf{3}$ Nature-instance-sequenceattribute-value-reference (N-ISAV-Ref) model. In this sense, the Nature field describes the nomenclature, the Instance field is analogous to the Entity, Attribute and Value fields retain the logic of its purpose, and the fields Sequence and Reference make possible the grouping of attributes of the entity. Analyzing this model, we can say that adding attributes to existing entities will not lead to changes in the database schema, which in turn will reflect the performance of applications and any communication between individual applications. Thus, this model should be appropriate when the legislation is undergoing changes and we want to minimize the negative consequences.

In such realized database, the description of the fields within the only table is implemented in the software part of the information system. Accordingly, the change in the data structure only leads to a change in the program code of the system, but not in the database schema. In this case this is a serious advantage because of the existence of dynamic data in the meaning of the system. 


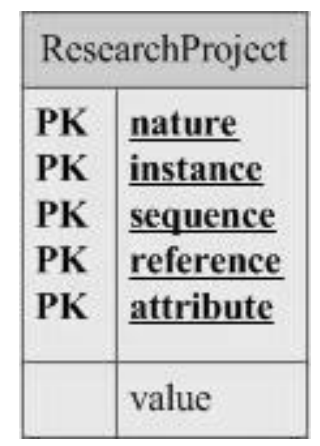

Figure 4. Data model for the dynamic part of the database

\section{CONCLUSIONS}

In the paper was proposed an alternative optimized schema of databases, which can be in use to the information systems with dynamic content. This schema (called N-ISAV-Ref) would be useful in terms of the easier integration between applications, which stands for quite a challenge, according to the dynamic situation in the modern world. Such a model would be appropriate for information systems that automate the actions of application, evaluation, negotiation and reporting of research projects. There are a lot of dynamic data within these operational stages. This dynamic data refers to data with variable structure and variable nature of different periods in terms of research projects. The paper focused on how we can reorganize the data in the system, so changes in the database due to changes in database structure to be minimal. The paper presented a method of storing data on the principle of attribute value, where the software part cares for the integrity of the data and the database takes care only for the storage.

\section{REFERENCES}

1. Krilova K., Electronic services in the public sector - state and trends, Economic and social alternatives, University of national and world economy - Sofia, Issue 4, 2014.

2. Kirilov R., Information Technologies for Career Development of University Staff at UNWE, 4th International Conference on Application of Information and Communication Technology and Statistics in Economy and Education (ICAICTSEE 2014), University of national and world economy, Sofia, 2014.

3. Frederic P. Miller, Agnes F. Vandome, John McBrewster, Entity-attribute-value Model: Data Model, Data Warehouse, Denormalization, Attribute- Value System, Linked Data, Resource Description Framework, Semantic Web, Inner- Platform Effect, Alphascript Publishing, 2010. 